\title{
Inverted bucket centrifugation with fluorinated oils and its applications to $T_{2}$ cut-offs
}

\author{
Ben Anger ${ }^{1, *}$, Stefan Hertel $^{2}$, Keith Love $^{3}$, Michael Ehiwario ${ }^{3}$, Matthias Appel ${ }^{1}$ \\ ${ }^{1}$ Shell Global Solutions International B.V., Shell Technology Centre Amsterdam, Amsterdam, Netherlands \\ ${ }^{2}$ Shell International Exploration and Production Inc., Shell Technology Center Houston, Houston, TX, USA \\ ${ }^{3}$ Shell Exploration \& Production Company, Shell Woodcreek, Houston, TX, USA
}

\begin{abstract}
Hydrocarbon reservoirs with a large column height as well as tight gas rocks require a large range of capillary pressures to describe the saturation of fluids present in these formations. While mercury injection capillary pressure (MICP) can achieve high equivalent capillary pressures, the tests are destructive to the core plugs. Centrifuge techniques have gained in popularity since they are faster than the porous plate technique, but they are limited in the achievable pressure range. Here, we propose the use of fluorinated oils to extend the achievable capillary pressure of the air-brine centrifuge technique by a factor of two. We use Fluorinert FC-70 in an inverted bucket configuration which doubles the radius of rotation and keeps the density contrast comparable to an air-brine system. Furthermore, we show the application to NMR $T_{2}$ cutoff determination as a function of capillary pressure. Since Fluorinert does not contain any hydrogen, there is no signal overlapping with the brine in the core plugs. Furthermore, in the inverted bucket configuration, the outlet face of the plug is not in contact with a drainage surface so that the Hassler-Brunner boundary condition of $\mathrm{Pc}=0$ is satisfied. Additionally, the method allows the storage under a liquid Fluorinert phase, which prevents evaporation and significantly extends the available time for NMR measurements at low water saturations.
\end{abstract}

\section{Introduction}

The interactions between wetting and non-wetting fluids in a porous rock are controlled in large part by capillary forces $[1,2,3,4]$. These forces determine fluid distributions and control the ease with which pore fluids can be recovered during production. The relationship between fluid saturations and capillary pressure is represented in capillary pressure curves. Lab measurements of these curves provide information on important petrophysical properties such as irreducible water saturation and residual oil saturation as well as insight into the initial hydrocarbon saturation in a reservoir. Reliable estimations of petroleum reserves depend on a complete understanding of the capillary pressure curves for a given reservoir.

Capillary pressure is defined as the pressure difference between the wetting and non-wetting phases as a function of the saturation of the wetting-phase in a porous medium. This pressure difference arises thanks to the interfacial tension that exists at the boundary between two immiscible fluids. Together with gravity, the capillary pressure in a porous medium controls both the distribution of fluid saturations as well as the ease with which fluids can move within the rock. As such, the capillary pressure distribution within a reservoir has a strong influence on the producible hydrocarbon volume.

Several methods exist to measure capillary pressure curves in porous rock through the displacement of the wetting fluid phase with a non-wetting phase [5]. Mercury intrusion capillary pressure (MICP) is a fast method for determining saturation as a function of capillary pressure, but rock samples are destroyed during MICP measurements. Steady-state displacement against a saturated porous plate provides an accurate measurement of saturations, but since the time required for equilibration at each capillary pressure can be on the order of several weeks, steady-state measurements are extremely time consuming.

A third common technique for the determination of capillary pressure curves is the use of a high-speed centrifuge to displace one reservoir fluid with another [6]. This method is non-destructive and is significantly faster than steady-state methods. However, accurate interpretation of high-speed centrifuge data is dependent on a series of assumptions that can lead to uncertainty [7]. Efforts have been made to address these inherent uncertainties using novel non-wetting fluids [8] or the use of magnetic resonance imaging. Green et al. [9],

* Corresponding author: benjamin.anger@shell.com 
combined the saturation gradient inherent in centrifuge measurements with one-dimensional NMR images to extract capillary pressure data from a sample that had been exposed to only one centrifuge speed $[9,10]$.

Proton magnetic resonance provides a powerful and non-destructive method for probing the plug saturation and fluid distributions within a rock sample. The combination of NMR methods and the high-speed centrifuge techniques allows for the determination of bound and free fluid volumes through the measurement of a quantity called the $T_{2}$ cut-off [11]. As illustrated in Figure 1, the $T_{2}$ cut-off is the boundary in $T_{2}$ space between movable and bound fluids at a given capillary pressure. NMR data from sample desaturated at a given capillary pressure is compared to the fully saturated NMR data to determine the $T_{2}$ cut-off.

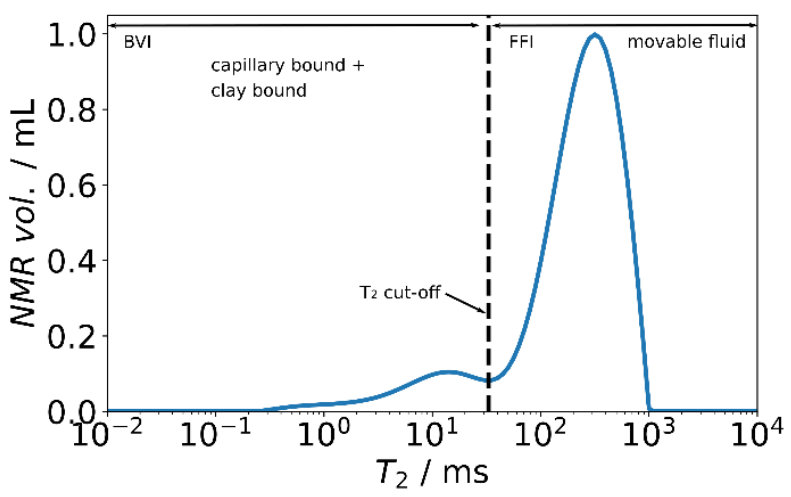

Figure 1. Fluid distribution and $T_{2}$ cut-off.

Generally, a single cut-off value is used as an input for NMR logging curves, which allows for separation of NMR $T_{2}$ distributions into the movable fluid fraction and the bound fluid fraction. However, it is not always clear which capillary pressure should be used to determine the irreducible water saturation and hence the $T_{2}$ cut-off value. It would therefore be desirable to develop a method to rapidly determine cut-off values at a range of capillary pressures.

In this work we present a method for rapid determination of movable and bound fluid volumes as a function of capillary pressure through a combination of NMR relaxometry and centrifuge primary drainage experiments in which formation brine is displaced by a dense fluorinated oil (Fluorinert FC-70). Because the nonwetting FC-70 is denser than the displaced brine, an inverted bucket configuration can be used in the centrifuge. The combination of comparable density contrast and the larger distance of the sample from the axis of rotation allows for desaturation at twice the capillary pressures compared to standard centrifuge methods. Further, in the inverted bucket configuration, the outlet face of the plug is not in contact with a drainage surface so the Hassler-Brunner boundary condition of $\mathrm{P}_{\mathrm{c}}=0$ is satisfied.

NMR $T_{2}$ measurements were made on a group of samples after multiple centrifuge speeds to produce $T_{2}$ cut-offs as a function of capillary pressure. This work showed that for this sample set, the commonly used cutoff of $33 \mathrm{~ms}$ should be replaced with a cut-off value of approximately $5 \mathrm{~ms}$ at the maximum capillary pressure achieved in the reservoir.

\section{Experimental methods}

\subsection{Samples}

The samples used in this work were sourced from a sandstone formation. All plugs were $2.54 \mathrm{~cm}$ in diameter. They were cleaned according to API RP 40 protocols, including UV fluorescence of solvent effluent and silver nitrate testing for salt content and then pressure saturated with $90 \mathrm{kppm} \mathrm{NaCl}$ equivalent synthetic formation brine. Klinkenberg-corrected permeability, helium porosity and MICP measurements were performed on twin plugs in a vendor lab. MICP saturations were calculated based on the normalization of the volume of $\mathrm{Hg}$ injected to the $\mathrm{He}$ porosity. Closure corrections and Hill-Shirley-Klein corrections were applied to correct for surface roughness and the presence of clay-bound water respectively. Sample details can be found in Table 1 .

Table 1. Sample details, $\mathrm{d}=2.54 \mathrm{~cm}$ for all plugs.

\begin{tabular}{|c|c|c|c|}
\hline Sample & $\begin{array}{c}\text { Klinkenberg } \\
\text { Perm. (mD) }\end{array}$ & $\begin{array}{c}\text { He porosity } \\
\text { (frac.) }\end{array}$ & $\begin{array}{c}\text { Length } \\
\text { (cm) }\end{array}$ \\
\hline C04 & 1.82 & 0.148 & 1.765 \\
\hline C05 & 2.43 & 0.178 & 1.602 \\
\hline C06 & 2.62 & 0.170 & 1.816 \\
\hline C07 & 2.96 & 0.174 & 2.034 \\
\hline C08 & 3.02 & 0.160 & 1.583 \\
\hline C09 & 3.26 & 0.158 & 1.836 \\
\hline
\end{tabular}

\subsection{Desaturation techniques}

\subsubsection{Steady-state displacement}

Several methods were tested for the displacement of brine with a fluorinated oil (Fluorinert FC-70.) Standard NMR $2 \mathrm{MHz}$ relaxometers are insensitive to $\mathrm{FC}-70$, so an experiment in which brine is displaced by a fluorinated oil provides maximum contrast. At any given capillary pressure, the only fluid present after the displacement that is visible in the NMR measurements is the brine remaining in the plug.

Steady-state displacement methods against a brinesaturated porous plate produces a uniform saturation distribution throughout the sample. While the results of these experiments are very robust, this type of desaturation can be extremely time consuming due to the very low flow rates typically associated with steady-state displacement. Our initial attempts were performed in hydrostatic Hassler-type core holders. These measurements were complicated by the fact that we were required to unload and reload each sample between each 
injection pressure in order to measure the brine saturation with NMR. During our initial attempts at this method, we determined that extraneous brine entered the samples during the process of unloading and loading as a result of the removal of the confining stress. This unavoidable ingress of brine added an unacceptable amount of uncertainty to the NMR brine volumes.

We then successfully performed a steady-state desaturation against a saturated porous plate in an NMR pressure cell. Since the core flood was performed in the NMR instrument, there was no need to remove the sample between subsequent desaturation steps. This effort led to generally satisfactory results, but several complications led us to abandon these efforts. The presence of the brinesaturated porous plate made precise determination of the saturation values at each injection pressure difficult since signal from the porous plate and core plug were combined in the NMR response. Separation of the two signal sources through subtraction is possible, but can lead to a significant decrease in signal to noise and an increase in uncertainty. Further, covering the full range of desired capillary pressures required more than two months for a single sample. The long measurement time combined with the fact that the NMR spectrometer was unavailable for other work during the experiment led us to seek alternative solutions.

\subsubsection{Centrifuge desaturation}

In order to achieve the desired range of capillary pressures on a realistic number of samples in an acceptable time frame, we turned to high-speed centrifuge displacement of the synthetic formation brine with FC-70. Two centrifuges were used in this work. The first was a Beckman Coulter Optima L-80XP Ultracentrifuge, and the second was a Beckman Coulter Optima L-90K. For all samples the inverted bucket orientation was used (see Figure 2), with the inlet face at a radius of $16.66 \mathrm{~cm}$ from the axis of rotation. Samples were centrifuged in sets of three or six simultaneously for 4-7 days per capillary pressure at rotation speeds of $1000,1500,2250,3200$, 3900, 5515, 10000 and 15000 RPM.

There are two main benefits to the use of the inverted bucket orientation. The distance of the plug from the axis of rotation is roughly double that of the radius in conventional buckets leading to a factor of two increase in the obtainable $P_{c}$ values. Further, no end piece is required with inverted buckets. Since the outlet face of the plug is not in contact with another surface, the HasslerBrunner boundary condition of $\mathrm{P}_{\mathrm{c}}=0$ at the outlet face is satisfied.

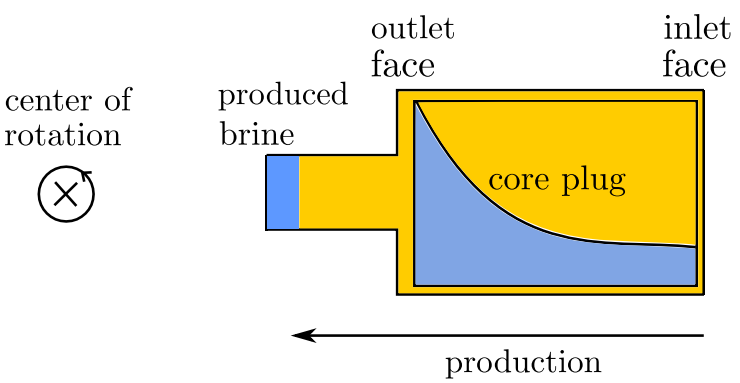

Figure 2. Inverted bucket orientation. In the plug, the remaining brine is blue and the FC-70 is yellow.

In the centrifuge, synthetic formation brine $(\rho=1.057$ $\left.\mathrm{g} / \mathrm{cm}^{3}, \eta=0.99 \mathrm{cP}\right)$ was displaced by FC-70 $(\rho=1.93$ $\left.\mathrm{g} / \mathrm{cm}^{3}, \eta=24 \mathrm{cP}\right)$. The change in weight of each plug was measured after each centrifuge speed to provide an independent measurement of the change in saturation at each capillary pressure. At all times during the series of measurements, the brine-saturated plugs were submerged in FC-70. This is important because the produced brine was never in contact with the plugs at any time, preventing brine from being imbibed into the samples and introducing uncertainty.

In order to correlate the $\mathrm{FC}-70 /$ brine capillary pressure dependent data with the $\mathrm{Hg}$ /air from the MICP measurements, we measured the contact angle of FC-70 in brine and the IFT to be $140^{\circ}$ and 55.47 dynes $/ \mathrm{cm}$ respectively. Each centrifuge speed produced a range of $\mathrm{Hg} /$ air equivalent capillary pressures dependent on plug length. A list of pressure ranges can be found in Table 2 .

\subsection{Nuclear magnetic resonance}

\subsubsection{Bulk NMR}

Bulk $T_{2}$ NMR measurements were performed on each sample after each centrifuge speed. Relaxation distributions were obtained via CPMG measurements at 2.3 MHz in Oxford Instruments GeoSpec2 relaxometers with $\mathrm{TE}=400 \mu \mathrm{s}$ (chosen to match common logging tool values), 12,500 echoes and a recycle delay of $7.5 \mathrm{~s}$. The NMR response of the samples were compared to a calibration sample of known water volume to calculate brine saturations. All NMR experiments were performed with the plugs submerged in a clean bath of NMR-silent FC-70. This provided the opportunity for extended signal averaging without the worry of the loss of brine through evaporation - an important consideration since the total brine volume present in plugs after high rotation speeds was on the order of $0.5 \mathrm{~cm}^{3}$ or less.

\subsubsection{D NMR imaging}

A uniform saturation distribution is not expected as a result of centrifuge desaturation experiments. To quantify this, one-dimensional saturation profiles were acquired at 12.7 MHz on an Oxford Instruments spectrometer using a simple frequency encode one-dimension profiling sequence. 


\section{Results and discussion}

All samples were centrifuged at the range of speeds described earlier. An example of the results for a single plug at all centrifuge speeds can be found in Figure 3. This plot shows a continued decrease in the total brine saturation as irreducible brine saturation is obtained at all capillary pressures. Further, the main peak of the $T_{2}$ distributions shifts to shorter times as brine in larger pores and more loosely bound brine is expelled from the sample.

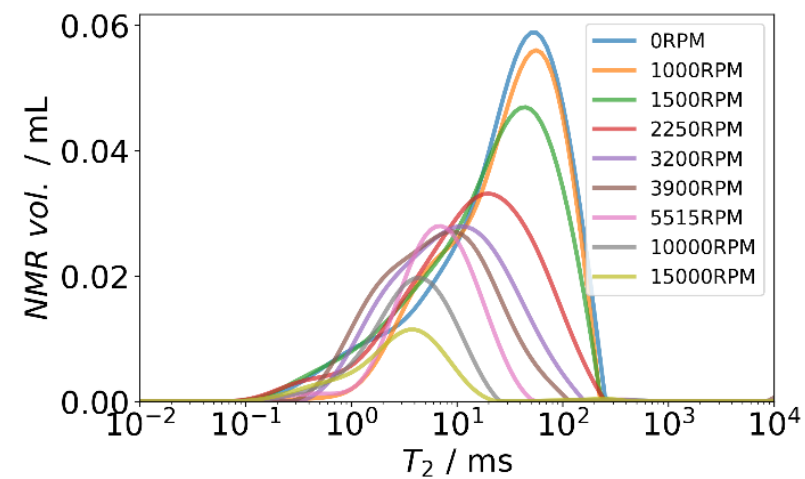

Figure 3. $T_{2}$ spectra for all capillary pressures for sample C09.

Since the synthetic brine was displaced by NMRsilent FC-70, the calibrated NMR volume after each rotation speed corresponds to the remaining water volume in the plug at a given capillary pressure and all movable brine has been displaced by Fluorinert. From this brine volume, a water saturation $S_{w}$ can be calculated for each plug as a function of capillary pressure. A comparison of NMR saturations to vendor-supplied MICP saturation data is shown in Figure 4. The Fluorinert-brine capillary pressures were averaged over the core plug and converted to the mercury/air capillary pressure through the wellknown conversion

$$
P_{H g a}=P_{F b} \frac{\sigma_{H g a} \cos \left(\theta_{\mathrm{Hga}}\right)}{\sigma_{F b} \cos \left(\theta_{F b}\right)}
$$

where $\sigma_{H g a}$ and $\sigma_{F b}$ are are the interfacial tensions for mercury/air and FC-70 brine and $\theta_{H g a}$ and $\theta F b$ are the contact angles for mercury/air and Fc-70/brine.

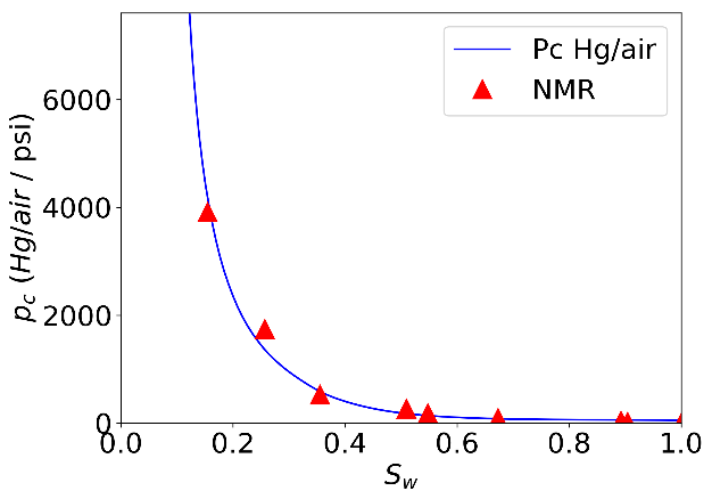

Figure 4. Comparison of NMR and MICP saturations for sample C09.
There is excellent agreement between the MICP and NMR data over the entire range of capillary pressures.

As seen in the comparison of NMR brine volumes to brine volumes calculated from the change in plug mass after centrifuging in Figure 5, there was good agreement between the two measurement methods.

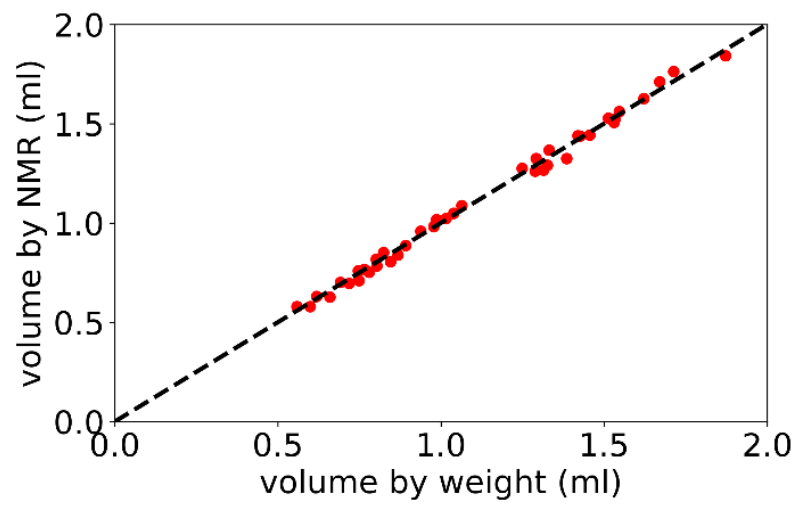

Figure 5. Comparison of NMR brine volume to calculated brine volume from change in sample mass.

A representative one-dimensional NMR saturation profile is shown in Figure 6. As expected $S_{w}$ approaches $100 \%$ at the outlet face.

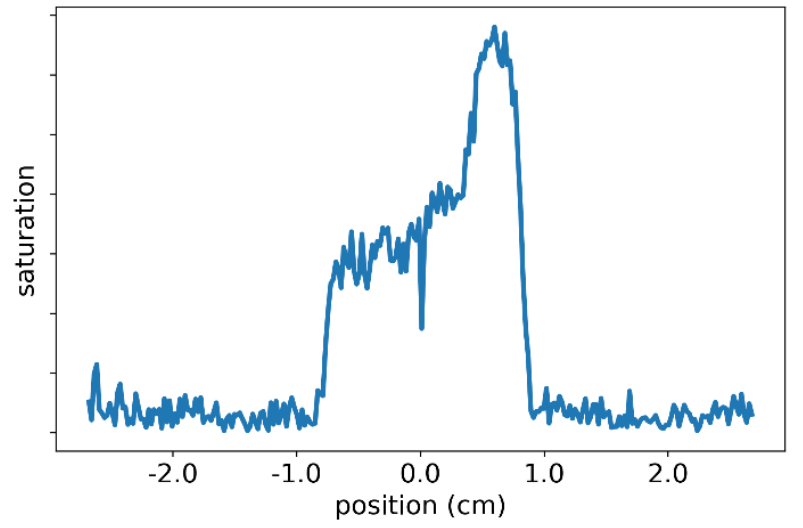

Figure 6. Saturation profile for sample C09 after centrifuging. Brine saturation approaches $100 \%$ at the outlet face on the right side of the plot.

As observed in the 1D NMR images, brine saturation in the plug after centrifugation is not uniform. If the Hassler-Brunner boundary condition of $\mathrm{P}_{\mathrm{c}}=0$ at the outlet phase is assumed, the variation in capillary pressure as a function of position along the plug is given by:

$$
P_{c}(r)=\frac{\Delta \rho \omega^{2}}{2}\left(R_{1}^{2}-r^{2}\right)
$$

where $\Delta \rho$ is the density contrast between FC-70 and the expelled brine, $\omega$ is the angular velocity of the centrifuge and $R_{l}$ is the distance of the inlet face from the axis of rotation. Given this distribution of capillary pressures and the one-dimensional profiles observed in several samples, an integrated average of the distribution described in equation 1 was calculated for each sample. The average $\mathrm{Pc}$ value is given by: 


$$
\bar{P}_{c}=\frac{\Delta \rho \omega^{2}}{2\left(R_{2}-R_{1}\right)^{2}} \int_{R_{1}}^{R_{2}}\left(R_{1}^{2}-r^{2}\right) d r
$$

where $R_{2}$ is the distance of the outlet face from the axis of rotation. A range of Pc values were calculated for each centrifuge speed and each sample used in this work. These values can be found in Table 2 .

Table 2. Equivalent FC-70/brine and $\mathrm{Hg}$ /air $\mathrm{P}_{\mathrm{c}}$ ranges for all centrifuge rotation rates.

\begin{tabular}{|c|c|c|}
\hline $\begin{array}{c}\text { Rotation Speed } \\
\text { (RPM) }\end{array}$ & $\mathbf{P}_{\mathbf{c}}$ (Fc-70/brine, psi) & $\mathbf{P}_{\mathbf{c}}$ (Hg/air, psi) \\
\hline 1000 & $3-5$ & $15-19$ \\
\hline 1500 & $7-10$ & $34-43$ \\
\hline 2250 & $18-22$ & $77-97$ \\
\hline 3200 & $36-45$ & $155-196$ \\
\hline 3900 & $54-68$ & $231-291$ \\
\hline 5515 & $108-134$ & $461-581$ \\
\hline 10000 & $348-442$ & $1515-1910$ \\
\hline 15000 & $786-995$ & $3410-4297$ \\
\hline
\end{tabular}

As shown in Figure 7, the $T_{2}$ cut-off at a given capillary pressure can be determined by comparing the cumulative NMR signal at $S_{w}=100 \%$ to the cumulative NMR signal from the desaturated sample. The $T_{2}$ cut-off is the value at which a horizontal line drawn through the final cumulative volume of the desaturated sample intersects with the cumulative volume of the fully saturated sample.

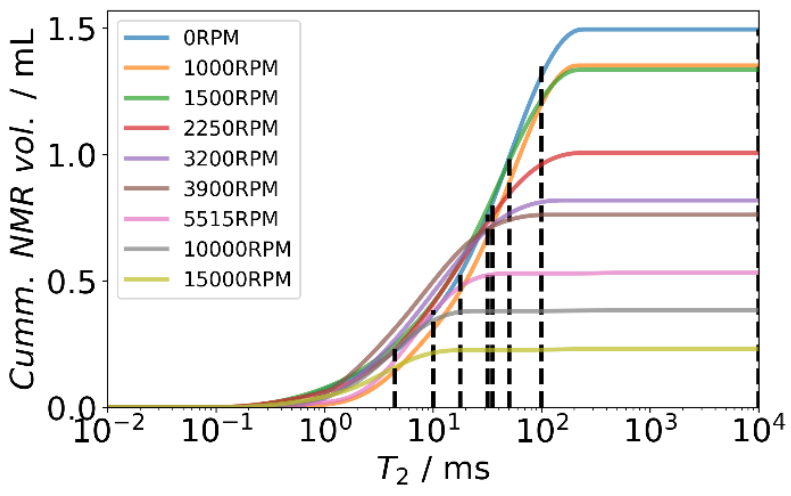

Figure 7. $T_{2}$ cut-off determination for sample C09.

Pressure dependent cut-off values were extracted for all samples. The results for sample $\mathrm{C} 09$ can be found in Figure 8. The commonly used sandstone cut-off of 33 $\mathrm{ms}$ is indicated by a dashed vertical line. While there is good agreement with the $\mathrm{C} 09$ data at a $\mathrm{Hg}$ /air equivalent of about 300 psi, it is clear that for higher capillary pressures the cut-off value between movable and bound fluid is much lower. For all samples in this work, the asymptotic $T_{2 i r}$ value was $4-5 \mathrm{~ms}$. In this case, use of the standard cut-off value would lead to large errors in the estimates of movable fluid volumes.

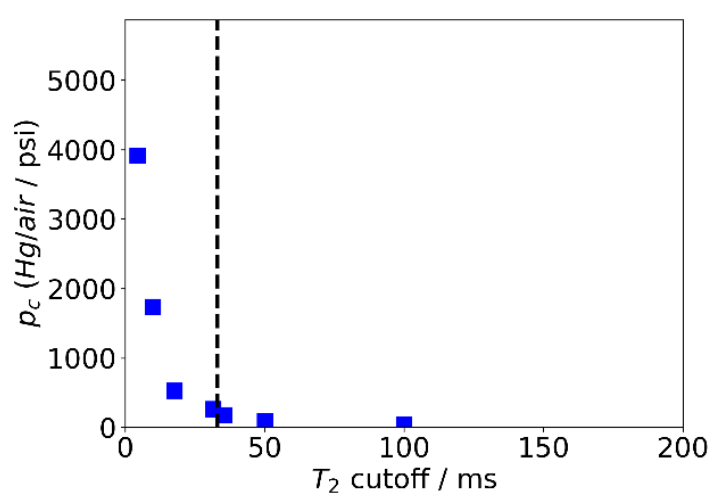

Figure 8. $T_{2}$ cut-off as function of Hg-air capillary pressure for sample C09. The dotted line is the standard $33 \mathrm{~ms}$ cut-off.

A modified Brooks-Corey model was applied to the data to extract asymptotic $T_{2}$ cut-off values for each sample. The standard Brooks-Corey [12] model relates capillary pressure to brine saturation as:

$$
P_{c}=P_{e}\left(\frac{S_{w 100}-S_{w i}}{S_{w}-S_{w i}}\right)^{n}
$$

where $P_{e}$ is the capillary entry pressure, $S_{w i}$ is the irreducible water saturation and $S_{w 100}$ (typically given as 1 ) is the maximum saturation. Following the recently published work of Al-Harbi et al. [13], this relationship can be recast in terms of relaxation times as:

$$
P_{c}=P_{e}\left(\frac{T_{2 \max }-T_{2 i r r}}{T_{2 c u t}-T_{2 i r r}}\right)^{n}
$$

where $S_{w 100}$ is replaced by $T_{2 \max }$ (longest $T_{2}$ value in the fully $T_{2}$ distribution of the saturated sample), $S_{w}$ is replaced by $T_{2 c u t}$ ( $T_{2}$ cut-off), and $S_{w i}$ is replaced by $T_{2 i r r}$ ( $T_{2}$ cut-off at irreducible saturation). $T_{2 \max }$ was between $300-400 \mathrm{~ms}$ for all samples.

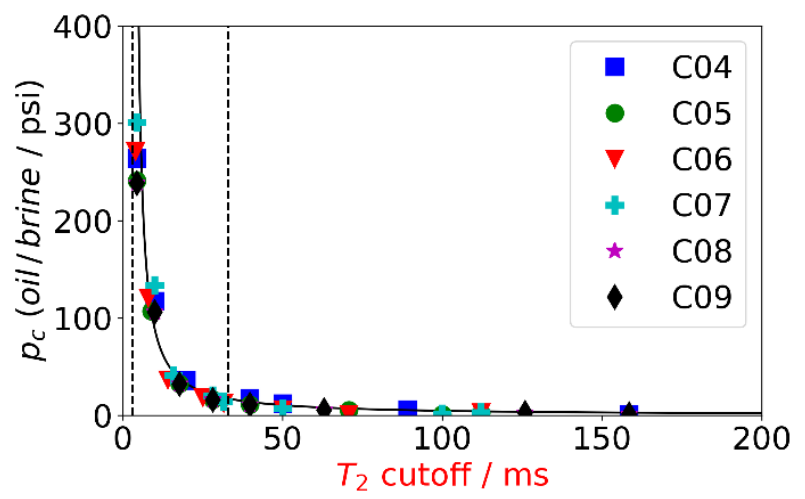

Figure 9. Brooks-Corey $T_{2}$ cut-off model applied to samples C04-C09 with $T_{2 i r r}=3.3 \mathrm{~ms}, P_{e}=1$ psi and $n=1.1$. Dashed lines are drawn at $T_{2 i r r}$ and at $33 \mathrm{~ms}$.

The application of the modified Brooks-Corey model to a selection of samples can be found in Figure 9. For all samples in this set, an asymptotic cut-off value in the range of 4-5 ms was found to provide a more accurate measure of the division between mobile and immobile pore fluids at irreducible water saturation at capillary 
pressures corresponding to those found in the reservoir from which the samples were obtained.

\section{Conclusions}

In this work we presented a novel method for quantifying movable fluid volumes as a function of capillary pressure through a combination of NMR relaxometry and primary drainage experiments in a centrifuge in which the formation brine is displaced by Fluorinert FC-70. The combination of a large density contrast between the wetting and non-wetting fluid phases and the increased distance of the sample from the axis of rotation allows for significantly larger capillary pressures compared to standard air-brine displacement measurements. This method allows for the storage and measurement of samples submerged in Fluorinert, which prevents evaporation and significantly extends the available time for NMR measurements at low saturations.

The samples measured in this work showed asymptotic cut-off values in the range of 4-5 ms, significantly shorter than the commonly used cut-off value of $33 \mathrm{~ms}$. The determination of a more accurate division between mobile and immobile fluid allows for more accurate estimates of movable fluids in place.

This work is directly applicable to reservoirs with large transition zones. In this specific case, it will be useful to produce a model in which the $T_{2}$ cut-off depends on capillary pressure and hence on the height above free water level. In a future paper, we will present the application of this method to the determination of a saturation model in which the $T_{2}$ cut-off varies as a function of the height above free water level.

Work is also currently underway to incorporate spatially resolved relaxation measurements of plugs after centrifuging using the 1D NMR profiling method developed by Hertel et al. [14]. This technique provides a snapshot of fluid distributions within a centrifuged plug on short time scale compared to standard 1D MRI methods. With this method, a significantly smaller number of centrifuge speeds will be needed to determine cut-off values at a full range of relevant capillary pressures.

The authors would like to thank Shell management for the permission to use the samples, and Kirk Smith, Aaron Dhaemers and Vivian Morah for their technical assistance.

\section{References}

1. J. Schön, Physical Properties of Rocks, Amsterdam: Elsevier, 2015.

2. E. Thomas, "Capillary Pressure Tutorial: Part 1 - It's a Jungle in Here," Petrophysics, vol. 59, no. 4, pp. 421-427, 2018.

3. E. Thomas, "Capillary Pressure Tutorial: Part 2 - The Path Out of the Jungle," Petrophysics, vol. 59, no. 5, pp. 557563, 2018.
4. E. Thomas, "Capillary Pressure Tutorial: Part 3 - The Jungle Gives Us Many Things," Petrophysics, vol. 59, no. 6, pp. 739-747, 2018.

5. C. McPhee, J. Reed and I. Zubizarreta, Core Analysis: A Best Practice Guide, Amsterdam: Elsevier, 2015.

6. G. Hassler and E. Brunner, "Measurement of capillary pressures in small core samples," Trans. of AIME, vol. 160, pp. 114-123, 1945.

7. D. O'Meara, G. Hirasaki and J. Rohan, "Centrifuge Measurements of Capillary Pressure: Part 1-Outflow Boundary Condition," SPE Reservoir Engineering, vol. 7, no. 1, pp. 133-142, 1992.

8. C. Straley and G. Leu, "Low-field NMR profiles for verification of oil and water saturations in cores," Proc. Soc. Core Anal., pp. SCA2006-31, 2006.

9. D. Green, J. Gardner, B. Balcom, M. McAloon and J. Cano-Barrita, "Comparison Study of Capillary Pressure Curves Obtained Using Traditional Centrifuge and Magnetic Resonance Imaging Techniques," in SPE Symposium on Improved Oil Recovery, Tulsa, OK, 2008.

10. Q. Chen and B. Balcom, "Measurement of rock-core capillary pressure curves using a single-speed centrifuge and one-dimensional magnetic-resonance imaging," $J$. Chem. Phys., vol. 122, p. 214720, 2005.

11. A. Timur, "Effective Porosity and Permeability of Sandstones Investigated Through Nuclear Magnetic Principles," The Log Analyst, vol. 10, no. 1, 1969.

12. R. Brooks and A. Corey, "Hydraulic Properties of Porous Media," Hydrology Papers, no. 3, 1964.

13. A. Al-Harbi, G. Hursan, H. Kwak and J. Gao, "A New NMR-based Height Saturation Model of a Low Permeability Carbonate Reservoir," Saudi Aramco Journal of Technology, pp. 49-59, 2018.

14. S. Hertel, D. de Kort, I. Bush, A. Sederman, L. Gladden, B. Anger, H. de Jong and M. Appel, "Fast spatiallyresolved T2 measurements with constant-gradient CPMG," Magnetic Resonance Imaging, vol. 56, pp. 70 76, 2019. 